\title{
Modelo multicapa para el estudio de difusión de fármacos en arterias a través de dispositivos intravasculares
}

\author{
Javier Escuer, Estefanía Peña, Miguel Ángel Martínez \\ Applied Mechanics and Bioengineering (AMB) \\ Instituto de Investigación en Ingeniería de Aragón (I3A) \\ Universidad de Zaragoza, Mariano Esquillor s/n, 50018, Zaragoza, Spain. \\ Tel.+34-976762707, e-mail:jescuer@unizar.es
}

\begin{abstract}
En el presente estudio se desarrolla un modelo numérico multicapa basado en una geometría 2D axisimétrica para describir el transporte convectivodifusivo de fármacos en el flujo sanguíneo y a través de la pared arterial.
\end{abstract}

\section{Introducción}

La aterosclerosis es una de las principales patologías que afecta a las arterias. Ésta consiste en la aparición de un depósito o placa compuesta por grasas, colesterol, calcio y otras sustancias que se encuentran en la sangre en la zona interna de la arteria. Con el tiempo esta placa crece y se endurece provocando el ralentizamiento del flujo sanguíneo e impidiendo que la sangre rica en oxígeno llegue a los órganos y a otras partes del cuerpo. En la actualidad, el stent metálico, generalmente recubierto de fármaco, se ha convertido en el principal dispositivo para el tratamiento de lesiones ateroescleróticas. El recubrimiento de estos dispositivos con sustancias antiproliferativas, como el paclitaxel o el sirolimus, pretende evitar la reestenosis: crecimiento de tejido en la zona lesionada tras la impantación del stent debido principalmente al daño mecánico ocasionado por el dispositivo. El uso de modelos computacionales en este caso aporta un mejor entendimiento de los factores físicos que influyen en los procesos de transporte de macromoléculas en los tejidos biológicos.

\section{Materiales y métodos}

Se presenta un modelo númerico multicapa basado en una geometría 2D axisimétrica correspondiente a un segmento recto de arteria. Está compuesto por siete regiones incluyendo lumen, endotelio, espacio subendotelial, lámina elástica interna, capa media, lámina elástica externa y adventicia. Los parámetros de transporte para las distintas capas han sido obtenidos de la literatura $[1,2,3]$.

El flujo sanguíneo, considerado como estacionario, laminar e incompresible, se ha descrito mediante las ecuaciones de Navier-Stokes. La pared arterial se ha considerado como un medio poroso heterogéneo, utilizando la Ley de Darcy para modelar el flujo transmural y para calcular la velocidad de filtración a través de las diferentes capas que la componen (espacio subendotelial, capa media y adventicia). Para describir el transporte de fármaco en el flujo sanguíneo se ha utilizado la ecuación de convección-difusión, mientras que el transporte de fármaco a través de la pared arterial se ha modelado mediante la ecuación de convección-difusiónreacción. El endotelio, la lámina elástica interna y lámina elástica externa han sido modeladas como membranas porosas semipermeables y el flujo a través de ellas ha sido descrito mediante las ecuaciones de Kedem-Katchalsky [4]. Además, se ha incorporado un modelo de "binding" no-lineal, reversible y saturable para describir la interacción entre el fármaco y las células musculares lisas (CML) existentes en la pared arterial [1,3].

Todas las ecuaciones presentadas han sido discretizadas mediante el método de los elementos finitos (MEF) utilizando el software comercial COMSOL Multiphysics 5.2 (COMSOL AB, Burlington, MA, USA).

Dos de los fármacos antiproliferativos más utilizados en la actualidad han sido estudiados y comparados. Finalmente, se han analizado distintas condiciones de liberación del medicamento desde el dispositivo (liberación rápida o lenta).

\section{Resultados}

Se muestran los resultados de la simulación en la zona de los dos primeros struts para diferentes tiempos después de la implantación del stent (Figura 1, izquierda). Se puede observar como para $t=1$ hora el fármaco ha invadido casi completamente la pared arterial. Se aprecia como la concentración es mayor en las zonas más cercanas a los struts y es relativamente menor en las zonas más próximas al lumen existentes entre los struts. Con el paso de los días, la distribución de la concentración se homogeneiza en la capa media. 
Con el objetivo de representar las predicciones del modelo desarrollado, se ha calculado también la concentración media normalizada del fármaco en las diferentes capas de la pared arterial. En la Figura 1 (derecha), se muestra la curva de concentración media normalizada de paclitaxel liberado por el stent en la capa media y en el espacio subendotelial. Se puede observar como después de un día de la implantación del stent, el nivel de concentración en la capa media es diez veces menor que el valor de concentración máxima. Después de una semana, el nivel de concentración es aproximadamente 100 veces menor.

\section{Conclusiones}

En este trabajo se ha propuesto un modelo numérico basado en la teoría del medio poroso y en datos obtenidos de la literatura con el que se ha estudiado los procesos de transporte de masa en el flujo sanguíneo y en las diferentes capas de la pared arterial.

Los resultados demuestran que el modelo presentado es válido para el estudio de la difusión de fármacos antiproliferativos en la arterial y puede ayudar al diseño de nuevos dispositivos liberadores de fármacos más eficientes.
Este trabajo ha sido posible gracias al Ministerio de Economía y Competitividad de España (MINECO), el cual financia esta investigación a través de la beca BES-2014-069737 y el proyecto DPI2013-44391-P.

\section{REFERENCIAS}

[1]. TZAFRIRI AR, LEVIN AD, EDELMAN ER (2009). Diffusion-limited binding explains binary dose response for local arterial and tumour drug delivery. Cell Proliferation 42(3):348-363.

[2]. MCGINTY S, MCKEE S, WADSWORTH RM, MCCORMICK C (2011) Modelling drug-eluting stents. Mathematical Medicine and Biology 28(1):129

[3]. BOZSAK, F, CHOMAZ, JM, BARAKAT, AI (2014). Modeling the transport of drugs eluted from stents: physical phenomena driving drug distribution in the arterial wall. Biomech. Model. Mechanobiol. 13(2), $327-347$.

[4]. KEDEM O, KATCHALSKY A (1958). Thermodynamic analysis of the permeability of biological membranes to non-electrolytes. Biochimica et Biophysica Acta 27:229-246.

\section{Agradecimientos}

\section{FIGURAS / LEYENDAS}
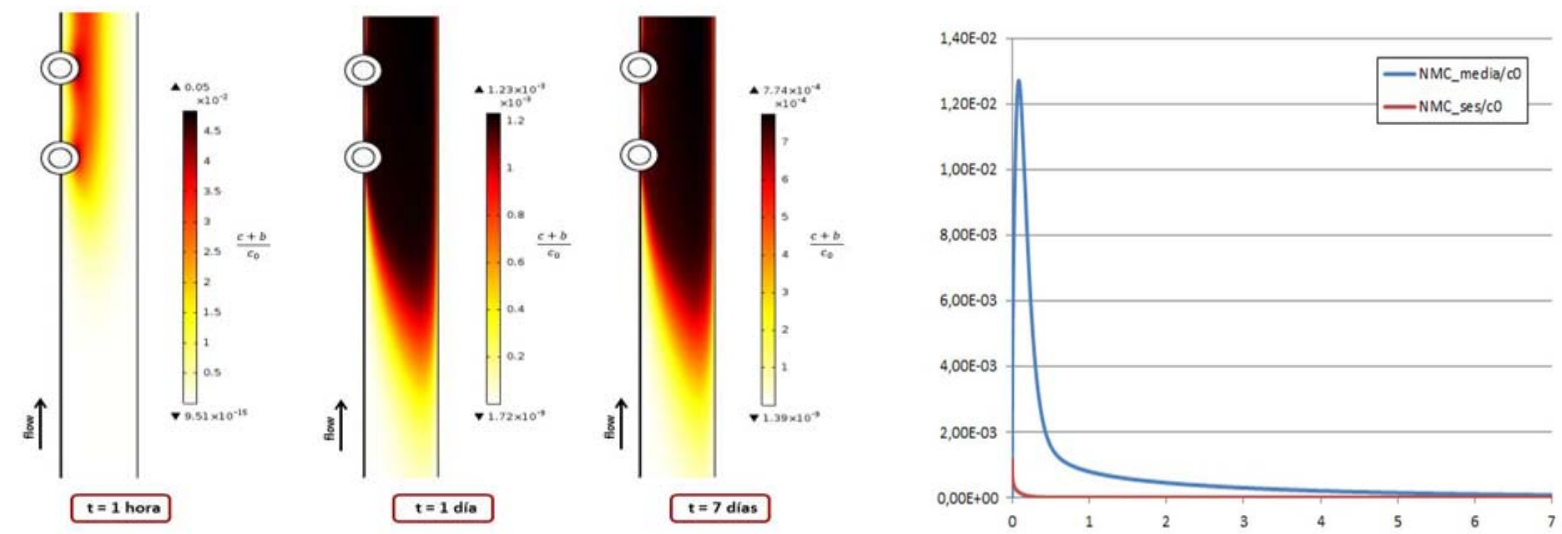

Figura 1. A la izquierda, concentración total (libre + ligado a CML) normalizada de paclitaxel en la capa media para diferentes tiempos después de la impantación del stent. A la derecha, curva de concentración media normalizada de paclitaxel liberado por el stent en la capa media y en el espacio subendotelial. 